\title{
Reactions at Wet-Dry Interfaces on Fibrous Materials
}

\author{
Robert Schaffer, Wm. D. Appel, and Florence H. Forziati
}

\begin{abstract}
The "brown-line" reaction on cellulosic materials at the boundary between areas wet with water and dry areas has now been found to be an example of a general phenomenon. Other fibers and liquids participate in oxidation reactions at wet-dry interfaces. Examples of the reactions are given, the conditions necessary for them are demonstrated, and the nature of the reactions is discussed.
\end{abstract}

\section{Introduction}

Bone and Turner $[1,2]{ }^{1}$ first reported that cellulose undergoes degradation at the wet-dry boundary upon continuous immersion of one end of a long strip of cotton cloth in water. This reaction was evidenced by the appearance of a brown line at the highest level to which water ascended. The color was found to result from the presence of a brown material that fluoresced in ultraviolet light and was water-soluble and reducing. When this material was washed away, the cloth itself was found to have been attacked with formation of an oxidized cellulose. As this oxidized cellulose was nontransportable and as it could be found only at the wet-dry boundary, the site of the reaction was established. Thus, the possibility was eliminated that the upward flow of water had concentrated the products of a reaction that occurred elsewhere in the wet region of the cloth.

Bogaty, Campbell, and Appel [3] confirmed the observation of Bone and Turner [2] that the oxidized cellulose was of the mildly reducing acid type and showed that among the soluble products were glucuronic acid, formic acid, and formaldehyde.

Although oxidation appears to be involved in the reaction, the identity of the oxidant has not been established. Bone and Turner [2] were unable to ascribe this role to peroxide or ozone, and as the reaction was found to occur in a nitrogen atmosphere as well as in air, atmospheric oxygen did not appear to be responsible. These workers recognized the possibility that oxygen held by the cotton might have accounted for the reaction observed under nitrogen. More recently, a partial inhibition of the reaction was reported by Madaras and Turner [4], who conducted experiments at a pressure of $8 \mathrm{~mm}$ of mercury and detected the formation of acidic oxidized cellulose at wet-dry interfaces but did not observe browning. To account for the formation of the oxidized cellulose, a Cannizzaro reaction was postulated involving terminal aldehyde groups produced by hydrolysis at the wet-dry interface. The brown material formed in experiments carried out in air was concluded to result from a secondary reaction involving oxygen.

The pronounced activity observed on cellulosic materials at the boundary between dry areas and areas wet with water made it of interest to investigate similar regions involving other liquids and other fibrous materials. Such studies have now been

Figures in brackets indicate the literature references at the end of this paper. made, the results of which indicate that the reaction at cellulose-water-air interfaces is an example of a general phenomenon. As this may be of wide interest, it seems desirable to report the results of the work at this time, even though many questions concerning the phenomenon remain to be answered.

\section{Materials and Methods}

The fibrous materials used in the investigation were cotton cloth (plain weave, commercially bleached, sizing-free, $3.6 \mathrm{oz} / \mathrm{yd}^{2}, 87$ warp yarns and 84 filling yarns per inch), nylon cloth (plain weave, $2.6 \mathrm{oz} / \mathrm{yd}^{2}, 158$ warp yarns and 96 filling yarns per inch), acetate cloth (plain weave, $13.3 \mathrm{oz} / \mathrm{yd}^{2}, 119$ warp yarns and 65 filling yarns per inch), and quartzfiber paper made by the Paper Section of the National Bureau of Standards. These materials were cut into strips, 1 to 2 in. wide, and purified.

The cotton strips were extracted with boiling 95 percent ethanol for $8 \mathrm{hr}$, immersed in boiling 1-percent sodium hydroxide solution in the absence of air for $2 \mathrm{hr}$, washed in distilled water, neutralized with dilute acetic acid, and again washed. The nylon strips were extracted with boiling ether for $6 \mathrm{hr}$, washed with a dilute solution of mild soap in distilled water, and thoroughly rinsed. The acetate strips were washed in dilute soap solution and thoroughly rinsed. The quartz-fiber paper was ignited at $800^{\circ}$ $\mathrm{C}$ for $16 \mathrm{hr}$.

The liquids used were benzene, xylene, chloroform, $n$-pentane, and distilled water. Of the organic liquids, the first three were reagent-grade chemicals that were distilled before use, the first two being previously dried over sodium. The $n$-pentane was of technical grade and was purified by shaking for 2 to $3 \mathrm{hr}$ with several portions of 30 -percent fuming sulfuric acid. It was then washed with water, dried over sodium wire, and distilled. The distilled water was redistilled first from acidified and then from alkaline permanganate.

The oxygen used was the usual commercial product; the nitrogen was a specially purified commercial product with an oxygen content below spectroscopic detectability.

Evaporation experiments were performed in Pyrex glass apparatus, which provided gas inlet and outlet tubes, and a support for the strip of fibrous material. The general arrangement was similar to that shown in figure 1. A sufficient length of fibrous material 


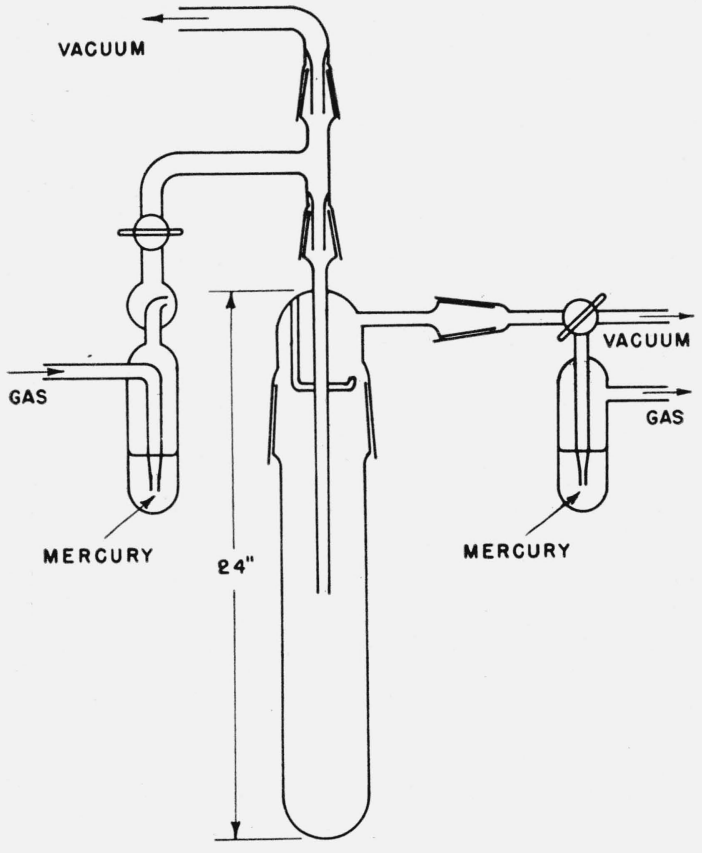

Figure 1. Apparatus used for experiments with n-pentane under nitrogen.

was suspended from the L-shaped support to reach the bottom of the chamber where it dipped into 200 to $300 \mathrm{ml}$ of the liquid to be evaporated. The flow of gas was adjusted to permit the liquid to rise by capillarity to between one-third and two-thirds of the height of the unimmersed portion of the fibrous strip. Under these conditions, several days were required for complete evaporation of the liquid. Usually, however, evaporations were not carried to dryness but were terminated when evidence of reaction, that is, fluorescence at the wet-dry interface, was detected. In the absence of evidence of reaction, experiments were continued for a minimum of 3 days. Some of the experiments were carried out in the diffuse light of the laboratory; others were carried out in a darkroom or were protected from light by wrapping the reaction chamber with heavy brown paper. Experiments were conducted at room temperature, which ranged between $23^{\circ}$ and $33^{\circ} \mathrm{C}$.

For evaporation of $n$-pentane under nitrogen, the apparatus shown in figure 1 was used. With the fibrous material installed, the apparatus was alternately evacuated on a high-vacuum line and flushed with nitrogen during a $40-\mathrm{hr}$ period. Simultaneously, approximately $300 \mathrm{ml}$ of $n$-pentane was degassed by usual techniques in another part of the vacuum system. Then, with the $n$-pentane frozen. and both parts of the system under vacuum, the stopcock between the two parts was opened and the $n$-pentane, as it warmed up, was allowed to distill into the chamber containing the fibrous material. The flow of gas was then begun and regulated at a rate sufficient to maintain the wet-dry interface at the desired height.

\section{Results and Discussion}

Table 1 presents a summary of the experiments.

When other fibrous materials and liquids were subjected to the conditions that favor brownline formation with cellulose and water, fluorescent lines visible under ultraviolet light were found at the wet-dry interfaces within 12 to $40 \mathrm{hr}$. Continuation of the experiments for several days resulted in visible browning at the interfaces. These results were obtained on cotton cloth upon evaporation of benzene (experiment 1), xylene (experiment 2 ), and chloroform (experiment 3); on acetate cloth and nylon cloth upon evaporation of water (experiments 7 and 8 , respectively); and on cotton cloth, nylon cloth, and quartz-fiber paper upon evaporation of $n$-pentane (experiments 4, 9, and 13, respectively).

The basis for this common pattern of behavior was sought in an investgation of conditions essential for the reactions.

As the reactions were obtained in experiments carried out in the absence of light (experiments $1,2,3$, and 13), it appears that the reactions are not photochemical in nature.

TABLE 1. Effect of continuous evaporation at room temperature of liquids from fibrous materials ${ }^{\mathrm{a}}$

\begin{tabular}{|c|c|c|c|c|}
\hline $\begin{array}{l}\text { Exper- } \\
\text { iment }\end{array}$ & $\begin{array}{l}\text { Fibrous } \\
\text { material }\end{array}$ & Liquid & Atmosphere & $\begin{array}{c}\text { Time when } \\
\text { fluorescence } \\
\text { was observed }\end{array}$ \\
\hline $1 \ldots$ & $\begin{array}{l}\text { Cotton, light } \\
\text { excluded. }\end{array}$ & & Oxygen ..... & Within $16 \mathrm{hr}$. \\
\hline 2 & _. do do _. . & Xylene & _... do ... & Do. \\
\hline 3 & _. do . . . . & Chloroform & $\ldots$ do $\ldots . . .$. & Within $12 \mathrm{hr}$. \\
\hline 4 & Cotton & $n$-Pentane... & $\ldots$ do _....... & Within $16 \mathrm{hr}$. \\
\hline 5 & $\begin{array}{l}\text { Cotton, en- } \\
\text { tire strip } \\
\text { wet, no in- } \\
\text { terface. }\end{array}$ & $\ldots$ do $\ldots$ & ..... do & $\begin{array}{l}\text { No fluores- } \\
\text { cence with- } \\
\text { in } 3 \text { days. }\end{array}$ \\
\hline $6 \ldots$ & $\begin{array}{l}\text { Cotton, de- } \\
\text { gassed for } \\
8 \text { days. }\end{array}$ & _..._do do . . & Nitrogen & Within $24 \mathrm{hr}$. \\
\hline & $\begin{array}{l}\text { Cellulose ace- } \\
\text { tate. }\end{array}$ & Water.... & Oxygen ..... & Within $40 \mathrm{hr}$. \\
\hline $\begin{array}{l}8 \\
9\end{array}$ & Nylon $\ldots . . . .$. & _... do ..... & _..... do . & $\begin{array}{l}\text { Do. } \\
\text { Within }\end{array}$ \\
\hline $10 \ldots$ & $\begin{array}{l}\text { Nylon, en- } \\
\text { tire strip } \\
\text { wet, no in- } \\
\text { terface. }\end{array}$ & ..... do & ...... do & $\begin{array}{l}\text { No fluores: } \\
\text { cence with- } \\
\text { in } 3 \text { days. }\end{array}$ \\
\hline 11. & Nylon...... & $\ldots$ do _............ & Nitrogen .... & Do. \\
\hline $13 \ldots$ & Quartz, light & $\begin{array}{l}n \text {-Pentane, shaken } \\
\text { with oxygen } 12 \\
\text { hr. and then de- } \\
\text { gassed. } \\
n \text {-Pentane........ }\end{array}$ & Oxygen ..... & Within $24 \mathrm{hr}$. \\
\hline $14 \ldots$ & Quartz & $\ldots$ do $\ldots$ & Nitrogen .... & $\begin{array}{l}\text { No fluores- } \\
\text { cence with- } \\
\text { in } 3 \text { days. }\end{array}$ \\
\hline
\end{tabular}

a Unless otherwise indicated, a strip of the fibrous material was suspended in the liquid in such a way that a wet-dry interface was present on the fibrous material. The fluorescence was observed at the interface. Experiments were carried out in the diffuse light of the laboratory except as indicated.

That these brown-line reactions are dependent upon the presence of contiguous wet and dry areas was suggested by experiments 5 and 10, using cotton and nylon with $n$-pentane in the presence of oxygen. Here, the entire lengths of the unimmersed portions of the fabrics were wet with ascending liquid throughout the 3 days of evaporation. This was accomplished by reducing the rate of flow of oxygen and employing shorter strips of cloth. With this arrangement, neither fluorescence nor browning were detected along the fabrics or upon the supporting rods. 
Evidence that oxygen is essential to the reactions was provided by experiments 11 and 14 , using $n$ pentane with nylon and quartz-fiber paper, respectively. These experiments were performed at atmospheric pressure in apparatus from which as much oxygen as possible was excluded. To do this, the fibrous material and liquid were degassed separately under high vacuum before being brought into contact. The evaporation of $n$-pentane for a 3-day interval in an atmosphere of prepurified nitrogen then occurred without detectable reaction. As reactions had been found to occur when oxygen was available during similar experiments (experiments 9 and 13), it became evident that oxygen was essential for the reaction. In view of the evidence (discussed below) that chemical modification of the fibrous material is not involved in the brown-line reaction that accompanies the evaporation of organic liquids, experiments 11 and 14 are believed to represent complete inhibition rather than limited reaction of the type reported by Madaras and Turner [4]. Oxygen thus appears to be of primary importance.

The likelihood that the fluorescence and brown material that resulted from evaporation of the organic liquids on fibrous materials were derived solely from chemical modification of the liquids, rather than from modification of the fiber, was suggested from the following observations:

1. The brown material obtained with $n$-pentane and cotton cloth (experiment 4) was soluble in $n$-pentane but not in water. Despite this difference in solubility from the brown material formed by evaporation of water from cellulose, both brown materials were capable of reducing silver thiosulfate in alkaline solution. These facts indicated that the $n$-pentane-soluble brown material was partially oxidized but capable of further oxidation, and in its solubility was unlike substances that might be expected from cellulose degradation.

2. After extraction of the brown material with $n$-pentane, the cotton cloth that had been used in the experiment with $n$-pentane (experiment 4) failed to show the reactions characteristic of oxidized celluloses with methylene blue and with alkaline silver thiosulfate solution, indicating that the cellulose had not been oxidized during the interface reaction.

3. The same lack of water-solubility of the brown products and the same nonreactivity of the extracted cotton to methylene blue and alkaline silver thiosulfate were observed in the experiments using benzene and xylene with cotton (experiments 1 and 2 ).

4. The brown-line reaction that accompanied the evaporation of $n$-pentane from quartz-fiber paper under oxygen (experiment 13) left little doubt that the liquid was the source of the brown products, in as much as quartz is not subject to oxidation.

In experiment 6 an extended degassing procedure was carried out on cotton cloth and n-pentane, which were then combined for evaporation in nitrogen. In contrast with similar experiments em- ploying nylon cloth and quartz-fiber paper with $n$-pentane (experiments 11 and 14), which gave no evidence of reaction, fluorescence was detected within $24 \mathrm{hr}$ after the start of evaporation. This result is not considered to contradict the conclusion. that oxygen is essential to the brown-line reaction with organic liquids, for, as suggested by Bone and Turner [2], it is possible that the cotton fibers may contain sufficient sorbed oxygen to account for the reaction. If, as suggested above, it is the $n$-pentane and not the fibrous material that undergoes chemical modification in the evaporation experiments, it is probable that the reaction will be essentially the same, regardless of the nature of the fibrous material. Thus, the fact that oxygen is essential to reaction during evaporation of $n$-pentane from nylon cloth and quartz-fiber paper suggests that it is also essential to reaction during evaporation of $n$-pentane from cotton cloth. In this connection, it is of interest that Madaras and Turner [4] observed no evidence of reaction when $n$-hexane and cyclohexane were evaporated from cotton cloth in a vacuum.

As both oxygen and wet-dry interfaces on the glass walls of the reaction chamber were available during many of the experiments described in this paper, evidence of the interface reaction should have appeared on the glass walls, if those conditions were sufficient to produce reaction. But in no experiment was evidence of reaction found anywhere except at the boundary between contiguous wet and dry areas on fibrous materials. Thus, even though the quantities of products formed are extremely small, wetdry boundaries on fibrous materials are to be regarded as constituting regions of enhanced chemical activity.

In order to show that the wet-dry interfaces on the fibrous materials did not merely serve as sites where products formed elsewhere were most readily detected, experiment 12 was conducted. In this experiment, $n$-pentane was distilled into the tube shown in figure 2, where it was shaken for $12 \mathrm{hr}$ in an atmosphere of oxygen and then degassed. The tube containing the $n$-pentane was then attached to the reaction. chamber in place of the vacuum connector shown at the top of figure 1, and the assembly, which contained a strip of nylon cloth, was degassed. The sealed capillary (fig. 2) was then broken, thus allowing the liquid to siphon in to the reaction chamber where the evaporation was conducted under nitrogen. Even with this most favorable arrangement for concentrating any products of the reaction of oxygen and $n$-pentane, there was no indication of fluorescence or of brown-line formation.

Evaporation of water from acetate cloth and nylon cloth in the presence of oxygen (experiments 7 and 8) demonstrated brown-line reactions with other combinations of materials. Here, as in the brown-line reaction with cellulose and water, the products are considered to be derived from the degradation of the fibrous materials. No attempt was made to verify this by experiment with the acetate and nylon. 


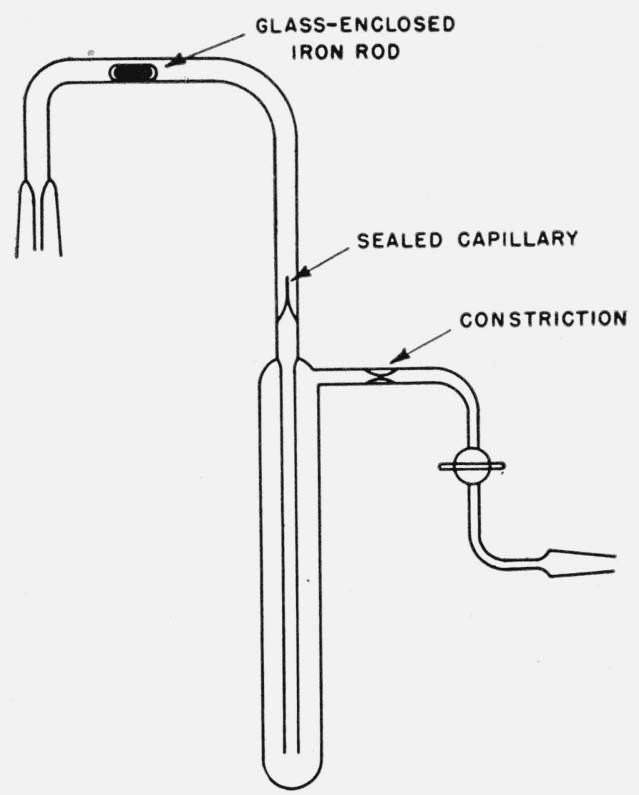

Figure 2, Tube in which n-pentane was shaken with oxygen.

\section{Conclusions}

The results suggest that continuous evaporation of liquid at a wet-dry interface on a fibrous material in contact with air is accompanied by chemical modification of either the liquid or fibrous material, depending on the kind of materials involved. With organic liquids, such as $n$ - pentane, reaction appears to be confined to the liquid; with water, modification of the fiber occurs. Characteristic of all of these reactions is the formation at the wet-dry interface of a brown line that fluoresces in ultraviolet light. Atmospheric oxidation is involved in at least some of the reactions, as shown by the fact that the brownline reaction occurring during the evaporation of $n$-pentane from nylon cloth and quartz-fiber paper in an oxygen atmosphere does not occur in a nitrogen atmosphere.

\section{References}

[1] W. A. Bone, J. Soc. Dyers Colourists 50, 307 (1934).

[2] W. A. Bone and H. A. Turner, J. Soc. Dyers Colourists 66, 315 (1950).

[3] H. Bogaty, K. S. Campbell, and W. D. Appel, Textile Research J. 22, 75 (1952).

[4] G. W. Madaras and H. A. Turner, J. Soc. Dyers Colourists 69, 371 (1953).

Washington, August 6, 1954 . 